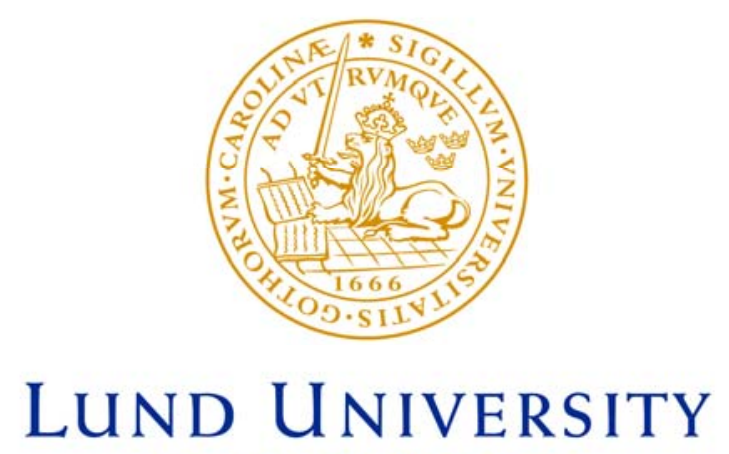

Faculty of Medicine

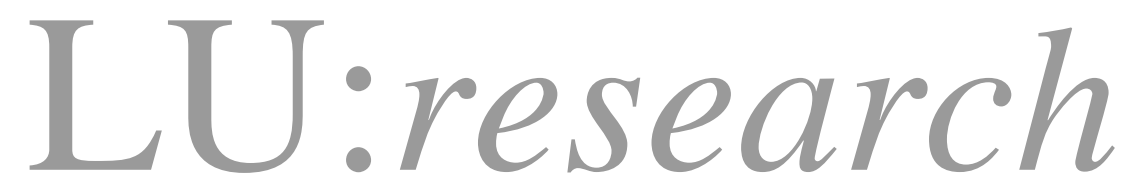

Institutional Repository of Lund University

This is an author produced version of a paper published in Alzheimer disease and associated disorders. This paper has been peer-reviewed but does not include the final publisher proof-corrections or journal pagination.

Citation for the published paper:

Bostrom, Fredrik and Jonsson, Linus and Minthon, Lennart and Londos, Elisabet.

"Patients with dementia with lewy bodies have more impaired quality of life than patients with Alzheimer disease"

Alzheimer Dis Assoc Disord, 2007, Vol: 21, Issue: 2, pp. 150-154.

http://dx.doi.org/10.1097NAD.0b013e318065c4a9

Access to the published version may require journal subscription.

Published with permission from: Wolters Kluwer 


\section{Patients with dementia with Lewy bodies have more impaired quality of life than patients with Alzheimer's disease.}

\section{Short Title: Quality of life in dementia with Lewy bodies}

Fredrik Boström $\mathrm{MD}^{1}$, Linus Jönsson MD MSc $\mathrm{PhD}^{2}$, Lennart Minthon MD $\mathrm{PhD}^{1}$, Elisabet Londos $\mathrm{MD} \mathrm{PhD}^{1}$

${ }^{1}$ Clinical Memory Research Unit, Department of Clinical Sciences Malmö, Lund University, Sweden

${ }^{2}$ European Health Economics AB, Stockholm, Sweden

Corresponding author:

Fredrik Boström

Lund University

Clinical Memory Research Unit, Deparment of Clinical Sciences Malmö

University Hospital MAS

se-205 02 Malmö, Sweden

Phone: +46 40334051

Fax: + 46334604

E-mail: fredrik.bostrom@skane.se

This study was financed by the Clinical Memory Research Unit, Department of Clinical Sciences Malmö, Lund University, Sweden

Disclosure: Dr Jonsson has served as consultant to the following companies: GE Healthcare, Sanofi-Aventis, Lundbeck, Pfizer, Neurochem, Elan and Wyeth. 


\begin{abstract}
AIM The primary aim of this study was to compare Quality Of Life (QoL) in patients with Dementia with Lewy Bodies (DLB) and patients with Alzheimer's Disease (AD). The secondary aim of this study was to investigate determinants of QoL in DLB.

METHODS 34 patients with DLB at the Neuropsychiatry clinic, University Hospital MAS, Malmö, Sweden, were included in a cross sectional study. These patients were matched to 34 patients with AD. Two QoL instruments, the EQ-5D instrument and the Quality of LifeAlzheimer's disease (QoL-AD) instrument, were applied in this study. Both instruments were administered to both patients and caregivers.

The DLB patients in this study have significantly lower QoL than the AD patients regardless of instrument or whether patient or caregiver-reported QoL was used. Furthermore this study shows that important determinants of quality of life in DLB include NPI score, independency in I-ADL, whether the patient is living with the caregiver and the presence of apathy and delusions.
\end{abstract}

Keywords: Dementia with Lewy bodies, DLB, Quality of life, QoL 


\section{Introduction}

Dementia is established as one of the major challenges of this century due to the enormous burden these pathologies impose on patients, caregivers and society. In subjects over 65 , crude prevalence rates for dementia in Europe vary between 5.9\% and 9.4\% with an exponential increase with age [1]. In two European population based studies, the prevalence of Dementia with Lewy Bodies (DLB) was estimated to 21.9\% (Finland) and 30.5\% (UK) of all dementia disorders [2]. DLB is today considered to be the second most common degenerative dementia disorder after Alzheimer’s disease (AD).

The core criteria of DLB are fluctuating cognition, visual hallucinations and spontaneous features of parkinsonism. Other typical symptoms of DLB are REM sleep behaviour disorder and severe neuroleptic sensitivity [3, 4]. Taking into account the difference in clinical manifestation of DLB and $\mathrm{AD}$, it is expected that the diseases differ in consequence for the patient, caregiver and society. This study focuses on Quality of Life (QoL) of DLB patients. In spite of an increasing interest in QoL studies, QoL research in AD is still considered to be in its infancy [5]. QoL in DLB is previously unstudied.

QoL is an elusive concept that has been defined and assessed in various ways. Health-related QoL is a somewhat narrower concept relating to the impact of physical and mental disorders and disability on the general well being of a person. One problem is that there is no gold standard when measuring QoL. In this study we use two widely used instruments, one generic and one specifically developed to assess QoL in patients with Alzheimer's disease (AD).

\section{Aim}

The primary aim of this study was to compare QoL in patients with DLB and patients with AD. The secondary aim of this study was to investigate determinants of QoL in DLB such as cognition, behavioural disorders, disability, age, co-morbidity, institutionalisation and whether the patient is living together with a caregiver or alone. 


\section{Methods and material}

\section{Study design}

34 patients with DLB attending regular visits at the memory clinic, University Hospital MAS, Malmö, Sweden, were prospectively interviewed according to the same protocol as $272 \mathrm{AD}$ patients, attending regular visits at six memory clinics in Sweden, Finland and Norway, earlier described by Jonsson et al [6]. From the 272 AD patients, 34 were selected to match the 34 DLB patients according to gender, age and cognitive function.

The matching was conducted by calculating a distance score between each possible pair of $\mathrm{AD}$-DLB patients with matching gender. The distance score was equal to by the weighted sum of the squared differences in age and MMSE scores (MMSE scores were given a 5-fold higher weight than age, to ensure groups were primarily matched with respect to MMSE scores). Patients were then matched by selecting the AD-DLB patient pairs with the lowest distance scores. (see table 1 )

The DLB diagnosis was confirmed using the revised DLB criteria [4]: 1) Progressive cognitive decline of sufficient magnitude to interfere with normal social or occupational. 2) At least two core features or one core feature and one suggestive feature have two be present. Core features are: Fluctuating cognition with pronounced variations in attention and alertness, recurrent visual hallucinations that are typically well formed and detailed, spontaneous features of parkinsonism. Suggestive features are: REM sleep behaviour disorder, severe neurloptic sensitivity, abnormally low uptake in basal ganglia on SPECT dopamine transporter scan, abnormally low uptake on MIBG myocardial scintigraphy. The AD patients fulfilled the ICD-10 criteria for Alzheimer's disease.

\section{Data collection procedure}

The examination of the DLB patients was designed be similar to the data collection procedure in the $\mathrm{AD}$ group[6].

The DLB and AD group were examined . in their homes or at a memory clinic together with their primary caregiver. 
The primary caregiver to the DLB patients were in 20 cases the spouse, in 11 cases the patient's child and in three cases a sibling. Cognitive function was assessed using MMSE [7]. Behavioural disturbances were measured using the Neuropsychiatric inventory (NPI) [8]. The DLB patients were in addition examined regarding dependency in Activities of Daily Living (ADL) using the Disability Assessment for Dementia scale (DAD) [9] which includes 40 items: 17 related to basic self-care and 23 to instrumental activities of daily living.

Co- morbidity was assessed in the DLB group using the same 12 domains used in the AD material. Information regarding prevalence of Parkinson's disease (PD) had been gathered in the AD material, but was not analysed as co-morbidity in this study as a PD diagnosis is in conflict with a DLB diagnosis.

\section{QoL instruments}

Two QoL instruments, the EQ-5D instrument [10] and the Quality of Life-Alzheimer's disease (QoL-AD) instrument [11], were applied in this study. Both instruments were administered to both patients and caregivers.

The EQ-5D is a generic QoL instrument in which the respondent is asked to rate their current health state on five dimensions (mobility, hygiene, usual activities, pain/discomfort and anxiety/depression), with three possible levels for each dimension.For each of the possible health states, a utility weight can be assigned through an algorithm that has been developed based on a time trade-off study in the United Kingdom [12]. The utility weight is a number $<1$ where 0 equals death and 1 equals perfect health, indicating the attractiveness of the health state based on the preferences of the general population. In contrast to the two other QoL scales used in this study, it is possible to obtain negative values of quality of life using this method. The EQ-5D also includes a visual analogue scale (VAS), anchored at perfect health (100) and death (0). Thus two values representing QoL are acquired using this instrument. Kendall's coefficient of concordance regarding EQ5D measurments has been estimated to $\mathrm{W}=0.984(\mathrm{p}<0.001)[10,13]$.

The QoL-AD is a QoL instrument specifically developed for use in patients with AD. Thirteen domains (physical health, energy, mood, living situation, memory, family, marriage, friends, self, ability to do chores, ability to do things for fun, money and life as a whole) are rated on a four-point scale, 1 being poor and 4 being excellent. The maximum score is 52 and 
the minimum 13. The Internal consistency reliability for QoL-AD ranges from 0.84 to 0.88 for patient and caregiver reported QoL in cognitively impaired patients[11, 14].

Patient and caregiver-reported QoL were analysed separately as this study focused on caregiver reported QoL.

\section{Statistical analysis}

For statistical analysis SPSS ${ }^{2}$ 12.0.1 was used. Spearman's rank order correlation was used to assess correlations between QoL-AD and the two values representing QoL that was acquired using EQ-5D as these values were not normally distributed. The Mann-Whitney Utest was employed to test for differences in QoL scores between groups. Two linear regression analyses were done on caregiver reported EQ-5D utility with an exclusion criterion for the examined determinants of $\mathrm{p}<0.10$. The first regression analysis included NPI score, independency in I-ADL, age, co-morbidity, institutionalisation and whether the patient is living with primary caregiver. The second regression analysis included all NPI items as separate variables.

\section{Results}

94\% of the DLB patients' caregivers and $97 \%$ of the AD patients' caregivers were able to complete both QoL instruments adequately. Corresponding figures for patient reports were $59 \%$ in the DLB group and $76 \%$ in the AD group.

Demographic data and assessed possible determinants of QoL in DLB and AD are presented in table 1. The DLB patients scored significantly higher on the brief NPI and were significantly more apathetic than the AD patients. The higher prevalence of hallucinations in the DLB group compared to the AD group was expected as this symptom is included in the DLB criteria.

Patient- and proxy-rated health-related QoL in DLB and AD are presented in table 2 and figure 1. Two AD patients and 8 DLB patients scored negative values on EQ-5D utility. The correlations between EQ-5D utility scores, EQ-5D VAS scale and QoL-AD for all patients (DLB and AD) are presented in table 3. 
Table 4 presents two regression models of NPI score, independency in I-ADL and whether the patient was living with the primary caregiver were found to be significant determinants of QoL in DLB in the first regression analysis. Apathy and delusions were found to be determinants of QoL in DLB in the second regression analysis (tab 4).

\section{Discussion}

This is to our knowledge the first study to describe quality of life in patients with Lewy-body dementia. The DLB and AD patients were not selected from the same population. There are some compatibility limitations to this method, however the same investigational protocol was used, thus the data produced was considered to be comparable.

The strong correlations that were found between three QoL measures (QoL-AD, EQ-5D utility and VAS scores) are in line with previous findings in Alzheimer's disease $[6,15,16]$. In this study, the EQ-5D questions, excluding the VAS scale, were easier to answer than the QoL-AD questions; only 5.9\% patients in the AD group and $29.4 \%$ of the patients in the DLB group were unable to complete the EQ-5D, compared to $32.4 \%$ of the $\mathrm{AD}$ patients and $35 \%$ of the DLB patients who were unable to complete the QoL-AD scale. The DLB patients were to a greater extent unable to answer questions regarding QoL adequately compared to the AD patients, although the DLB and AD patients were matched regarding cognitive function. It was not the aim of this study to investigate the reason for this difference, however one may speculate that the greater prevalence of apathy in the DLB group may play an important role. Another possible contributing factor is poorer conversational skills among DLB-patients as reported by Ferman et al [17]. This result implicates the limits of trying to assess the general level of ability in dementia using only measurements of cognitive function.

The focus of this study is on caregiver reported QoL as patient-reported QoL has been shown not to correlate with cognition or progression of the disease in patients with dementia [15] [18], even though most healthy subjects would probably strongly prefer a health state with intact cognitive function to a state with severe cognitive impairment. The caregivers may be in a better position to provide estimates that reflect how patients might have interpreted their QoL had they been cognitively intact. Caution must however be taken when interpreting caregiver reported QoL, as it is not only affected by factors concerning state of the patients, but also by factors concerning the caregiver, such as caregiver burden and caregiver 
depression [19, 20]. As in several previous studies, we found that caregiver-reported QoL was considerably lower than patient-reported QoL for all measurements $[6,11,16,19]$.

Whether to use proxy reported QoL in patients with dementia is a debated issue.However, in this study the DLB patients have significantly lower QoL than the AD patients regardless of whether patient or caregiver -reported QoL was used. . A possible reason for this difference is a higher prevalence of behavioural disorders in the DLB group compared to the AD group. Our clinical impression is that apathy is particularly stigmatising and prevalent in patients with DLB. This impression is supported by apathy being significantly more prevalent in the DLB group compared to the AD group, and also that apathy was associated with lower QoL within the DLB group in the multiple regression analysis. Another possible explanation of the difference in QoL in these cognitively matched DLB and AD groups is that a decreased cognitive level signals farther progression of the disease in DLB than it does in AD.

In $6 \%$ of the $\mathrm{AD}$ patients and $24 \%$ of the DLB patients the caregiver-rated EQ-5D scores corresponded to below-zero utility values according to the UK scoring algorithm. This indicates that the general population sample used to obtain the scoring algorithm considered these health states to be worse than death. The finding that almost one in four patients with DLB are in health states considered equal to or worse than death is alarming. The corresponding figure for the AD group was six percent, markedly less compared to the DLB group.

We found that NPI score, dependency in I-ADL and whether the patient was living with the caregiver were significant determinants of QoL in DLB. NPI score and whether the patient was living with the caregiver were also found to be determinants for QoL in the complete AD group ( $\mathrm{n}=272$ ) whereas I-ADL was not assessed in the AD study [6]. ADL and I-ADL have however been shown to be determinants of QoL in dementia in other studies [21, 22] .

Apathy and delusions were the only NPI items that were individually significant determinants of QoL in DLB. Earlier studies indicate that apathy may be a general determinant of QoL in dementia [23]. Delusions were not as prevalent in the DLB group as apathy, but may be a specific determinant of QoL in DLB. There are no earlier reports of delusions as a determinant of QoL in dementia. 
Cognitive function was not a determinant of QoL in DLB in this study. Cognitive function as a determinant of QoL in DLB has not previously been studied. In other dementias, cognitive function correlates with QoL in some studies [15] [24], but not in others [6] [22].

The results of this study and an earlier study of resource utilisation in DLB vs in AD[25], indicate that the consequences of DLB and AD differ greatly; a DLB diagnosis predicts an almost threefold increase in resource utilisation and a significantly lower QoL compared to AD. These results underline the importance making a correct differential diagnosis of degenerative dementia, as the diagnosis is likely to influence the gravity and type of problems that will have to be addressed during the course of the disease.

The QoL in caregivers to patients with DLB was not studied here, but should be examined in future studies as it is affected in other dementia disorders [26, 27].

In order to examine if the extensive impairment of QoL in DLB is reversible, interventional studies that include QoL as an outcome measures are needed.

In summary the results of this study suggest important differences in quality of life between patients with DLB and AD, and that important determinants of quality of life in DLB include NPI score, independency in I-ADL, whether the patient is living with the caregiver and the presence of apathy and delusions.

The present study is limited by a small sample size. More research is needed to confirm the findings of this study and to further examine other possible determinants of QoL in DLB such as motor dysfunction and autonomic dysfunction.

\section{Aknowledgements}

Eva Falk for administrative support.

Roberta Boson for linguistical support.

This research was supported by Alzheimer Foundation, Sweden 


\section{References}

1. Berr C, Wancata J, and Ritchie K, Prevalence of dementia in the elderly in Europe. Eur Neuropsychopharmacol, 2005. 15(4): p. 463-71.

2. Zaccai J, McCracken C, Brayne C, A systematic review of prevalence and incidence studies of dementia with Lewy bodies. Age an Agening, 2005. 34: p. 561-566.

3. McKeith IG, Galasko D., Kosaka K, et al. Consensus guidelines for the clinical and pathologic diagnosis of dementia with Lewy bodies (DLB): report of the consortium on DLB international workshop. Neurology, 1996(47): p. 1113-24.

4. McKeith IG, Dickson DW, Lowe J, et al. Diagnosis and management of dementia with Lewy bodies. Neurology, 2005. 65 (12): p. 1863-1872.

5. Whitehouse PJ, Patterson MB, and Sami SA, Quality of life in dementia: ten years later. Alzheimer Dis Assoc Disord, 2003. 17(4): p. 199-200.

6. Jonsson L, Andreasen N, Kilander L, et al. Patient- and proxy-reported utility in Alzheimer disease using the EuroQoL. Alzheimer Dis Assoc Disord, 2006. 20(1): p. 49-55.

7. Folstein MF, Folstein SE., McHugh PR, "Mini-mental state". A practical method for grading the cognitive state of patients for the clinician. J Psychiatr Res., 1975. 12: p. 189-198.

8. Cummings JL, Mega M., Gray K, et al. The Neuropsychiatric Inventory: comprehensive assessment of psychopathology in dementia. Neurology, 1994. 44(12): p. 2308-14.

9. Gelinas I, Gauthier L., McIntyre M, et al. Development of a functional measure for persons with Alzheimer's disease: the disability assessment for dementia. Am J Occup Ther., 1999. 53(5): p. 471-81.

10. The EuroQol Group. EuroQol--a new facility for the measurement of health-related quality of life. Health Policy, 1990. 16(3): p. 199-208. 
11. Logsdon RG, GibbonsLE., McCurry SM, et al. Assessing quality of life in older adults with cognitive impairment. Psychosom Med, 2002. 64(3): p. 510-9.

12. Dolan P, Modeling valuations for EuroQol health states. Med Care, 1997. 35(11): p. 1095-108.

14. Ready RE, Ott BR. Quality of Life measures for dementia. Health Qual Life Outcomes, 2003. 1(1): p. 11.

15. Thorgrimsen L, Selwood A, Spector A, et al. Whose quality of life is it anyway? The validity and reliability of the Quality of Life-Alzheimer's Disease (QoL-AD) scale. Alzheimer Dis Assoc Disord, 2003. 17(4): p. 201-8.

16. Naglie G, Tomlinson G, Tansey C, et al. Utility-based Quality of Life Measures in Alzheimer's Disease. Qual Life Res, 2006. 15(4): p. 631-43.

17. Ferman TJ, Smith GE, Boeve BF, et al., Neuropsychological differentiation of dementia with Lewy bodies from normal aging and Alzheimer's disease. Clin Neuropsychol, 2006. 20(4): p. 623-36.

18. Selwood A, Thorgrimsen L, Orrell M. Quality of life in dementia--a one-year followup study. Int J Geriatr Psychiatry, 2005. 20(3): p. 232-7.

19. Sands LP, Ferreira P, Stewart AL, et al. What explains differences between dementia patients' and their caregivers' ratings of patients' quality of life? Am J Geriatr Psychiatry, 2004. 12(3): p. 272-80.

20. Karlawish JH, Casarett D, Klocinski J, et al. The relationship between caregivers' global ratings of Alzheimer's disease patients' quality of life, disease severity, and the caregiving experience. J Am Geriatr Soc, 2001. 49(8): p. 1066-70.

21. Andersen CK, Wittrup-Jensen KU, Lolk A, et al. Ability to perform activities of daily living is the main factor affecting quality of life in patients with dementia. Health Qual Life Outcomes, 2004. 2: p. 52. 
22. Wlodarczyk JH, Brodaty H, Hawthorne G. The relationship between quality of life, Mini-Mental State Examination, and the Instrumental Activities of Daily Living in patients with Alzheimer's disease. Arch Gerontol Geriatr, 2004. 39(1): p. 25-33.

23. Samus QM, Rosenblatt A, Steele C, et al. The association of neuropsychiatric symptoms and environment with quality of life in assisted living residents with dementia. Gerontologist, 2005. 45 Spec No 1(1): p. 19-26.

24. Banerjee S, Smith SC, Lamping DL, et al. Quality of life in dementia: more than just cognition. An analysis of associations with quality of life in dementia. J Neurol Neurosurg Psychiatry, 2006. 77(2): p. 146-8.

25. Bostrom F, Jonsson L, Minthon L, Londos E. Patients with Lewy body dementia use more resources than those with Alzheimer's disease. Int J Geriatr Psychiatry, 2006. In press.

26. Kurz X, Scuvee-Moreau J, Vernooij-Dassen M, et al. Cognitive impairment, dementia and quality of life in patients and caregivers. Acta Neurol Belg, 2003. 103(1): p. 2434.

27. Argimon JM, Limon E, Vila J, et al. Health-related quality-of-life of care-givers as a predictor of nursing-home placement of patients with dementia. Alzheimer Dis Assoc Disord, 2005. 19(1): p. 41-4. 
Table 1 Between-group comparisons of demographic data, co-morbidity, and test score results

\begin{tabular}{|c|c|c|c|}
\hline & $\begin{array}{c}\text { DLB } \\
\text { mean(range) }\end{array}$ & $\begin{array}{c}\mathrm{AD} \\
\text { mean(range) }\end{array}$ & $\mathrm{p}$ for difference \\
\hline$N$ & 34 & 34 & \\
\hline Gender (male/female) & $19 / 15$ & $19 / 15$ & 1 \\
\hline age & $77.4(64-87)$ & $78.2(63-92)$ & 0.92 \\
\hline Co- morbidity & $0.88(0-3) \wedge$ & $1.06(0-4) \wedge$ & 0.752 \\
\hline Hypertension(n) & 12 & 11 & - \\
\hline Heart failure(n) & 4 & 6 & - \\
\hline Thyroid disease(n) & 4 & 1 & - \\
\hline Diabetes(n) & 3 & 3 & - \\
\hline Autoimmune disorder(n) & 2 & 0 & - \\
\hline Cancer(n) & 2 & 2 & - \\
\hline Hyperlipidemia(n) & 2 & 3 & - \\
\hline Ischemic heart disease(n) & 1 & 4 & - \\
\hline Stroke(n) & 0 & 2 & - \\
\hline Asthma(n) & 0 & 1 & - \\
\hline $\operatorname{COPD}(n)$ & 0 & 1 & - \\
\hline Migraine(n) & $\begin{array}{c}0 \\
17,3(0-29)\end{array}$ & $\begin{array}{c}2 \\
16.9(0-30)\end{array}$ & 0.92 \\
\hline \$NPI-brief (max 30) & $6.29(0-17) \S$ & $3.50(0-11) \S$ & $0.048 *$ \\
\hline Delusions & $0.62 \pi$ & $0.50 \pi$ & 0.622 \\
\hline Hallucinations) & 1.06 & 0.26 & $<0.001^{* * *}$ \\
\hline Agitation/aggression & 0.299 & 0.35 & 0.371 \\
\hline Dysphoria & $0.62 \pi$ & 0.35 - & 0.233 \\
\hline Anxiety & $0.74 \rrbracket$ & $0.53 \rrbracket$ & 0.411 \\
\hline Euphoria & $0.03 \pi$ & $0.03 \pi$ & 0.966 \\
\hline Apathy & $1.26 \pi$ & $0.68 \pi$ & $0.021^{*}$ \\
\hline Desinhibition & 0.15 & 0.09 - & 0.727 \\
\hline Irritability/labilit) & $0.24 \uparrow$ & 0.419 & 0.206 \\
\hline Aberrant motor activity & 0.419 & 0.299 & 0.503 \\
\hline DAD & $15.03(0-37)$ & not done & - \\
\hline B-ADL & $8.88(0-20)$ & not done & - \\
\hline I-ADL & $6.15(0-18)$ & not done & - \\
\hline \& Caregiver living with patient & 15 & 22 & \\
\hline \# Special living & 15 & 4 & \\
\hline
\end{tabular}

All results are presented as mean (range) if not otherwisely stated. Matched variables. \$ A brief version of the NPI. § Total brief NPI results. ^ Severity of each symptom (range 0-3). ^ Co morbidity added to a single variable.£Number of patients living with their primary caregiver. \# Number of patients with assisted living such as service flats, group living or nursing home. 
Table 2 Patient- and caregiver-rated health-related quality of life. (mean +/- standard deviation)

DLB

AD Difference (p)\# Difference (p) ${ }^{a}$

EQ-5D utility

$\%$ completed, patient (n)

$\%$ completed, caregiver (n)

$71(24)$

$100(34)$

94(32)

Patient-rated utility

$0.38+/-0.38$

100(34)

Caregiver-rated utility

$0.24+/-0.30$

$0.87+/-0.17$

$0.56+/-0.29$

$<0.0001$

$<0.0001$

$<0.0001$

$<0.0001$

\section{VAS scale}

\% completed, patient 65(22) 79(27)

\% completed, caregiver

Patient-rated utility

Caregiver-rated utility

$$
100 \%
$$

$55+/-17$

97(33)

$43+/-22$

$76+/-19$

$53+/-20$

0.0002

0.001

0.037

0.22

\section{QoL-AD}

$\%$ completed, patient*

$\begin{array}{rr}68(23) & 88(30) \\ 94(32) & 100(34) \\ 2.29+/-0.41 & 2.89+/-0.45 \\ 2.01+/-0.35 & 2.35+/-0.45\end{array}$

$<0.0001$

0.0001

Patient-rated utility

$2.01+/-0.35$

$2.35+/-0.45$

0.002

0.012

*Counted as completed if 1 or less questions missing.

\#Using Mann Whitney U-test

a Corrected for multiple comparison (Bonferroni) 
Table 3 Pearson correlation of used QoL instruments in all studied patients

\begin{tabular}{lllllll}
\hline & $\begin{array}{l}\text { EQ-5D } \\
\text { (patient) }\end{array}$ & $\begin{array}{l}\text { VAS } \\
\text { (patient) }\end{array}$ & $\begin{array}{l}\text { QoL-AD } \\
\text { (patient) }\end{array}$ & $\begin{array}{l}\text { EQ-5D } \\
\text { (caregiver) }\end{array}$ & $\begin{array}{l}\text { VAS } \\
\text { (caregiver) }\end{array}$ & $\begin{array}{l}\text { QoL-AD } \\
\text { (caregiver) }\end{array}$ \\
\hline $\begin{array}{l}\text { EQ-5D } \\
\text { (patient) }\end{array}$ & 1 & $0.49(0.11)^{* *}$ & $0.56(0.10)^{* *}$ & $0.48(0.10)^{* *}$ & $0.109(0.13)$ & $0.30(0.12)^{*}$ \\
$\begin{array}{l}\text { VAS } \\
\text { (patient) }\end{array}$ & 1 & $0.59(0.10)^{* *}$ & $0.45(0.11)^{* *}$ & $0.33(0.13)^{*}$ & $0.40(0.12)^{* *}$ \\
$\begin{array}{l}\text { QoL-AD } \\
\text { (patient) }\end{array}$ & & 1 & $0.51(0.10)^{* *}$ & $0.207(0.13)$ & $0.56(0.10)^{* *}$ \\
$\begin{array}{l}\text { EQ-5D } \\
\text { (caregiver) }\end{array}$ & & & 1 & $0.60(0.08)^{* *}$ & $0.64(0.07)^{* *}$ \\
$\begin{array}{l}\text { VAS } \\
\text { (caregiver) }\end{array}$ & & & & & & $0.60(0.08)^{* *}$ \\
$\begin{array}{l}\text { QoL-AD } \\
\text { (caregiver) }\end{array}$ & & & & & & 1 \\
\hline
\end{tabular}

Correlations are presented as r(SE)

** Correlation is significant at the 0.01 level (2-tailed).

* Correlation is significant at the 0.05 level (2-tailed).

Grey cells represent comparisons of patient and caregiver reported quality of life 
Table 4. Linear regression analyses of proxy rated EQ-5D utility in DLB

\begin{tabular}{lcc}
\hline & Model 1 & Model 2: NPI items \\
\hline Exclude variables & $\begin{array}{c}\text { MMllucinations, Agitation, } \\
\text { living, total morbidity }\end{array}$ & $\begin{array}{c}\text { Depression, Anxiety, Euphoria, } \\
\text { Desinhibition, Irritability, } \\
\text { Aberrant motor behaviour, Sleep } \\
\text { disorder, Eating disorder }\end{array}$ \\
\hline $\mathrm{N}$ & $\mathbf{3 4}$ & $\mathbf{3 4}$ \\
\hline NPI & $\mathbf{B ~ ( \mathbf { p } )}$ & $\mathbf{B ~ ( p )}$ \\
I-ADL & $-0.008(0.003)$ & - \\
Living with patient & $0.019(0.015)$ & - \\
\# Delusions & $0.161(0.042)$ & - \\
\# Apathy & - & $-0.035(0.018)$ \\
(constant) & - & $-0.037(0.001)$ \\
$\mathrm{R}^{2}$ & $0.198(0.035)$ & $0.472(<0.001)$ \\
\hline
\end{tabular}

Two linear regression analyses where obtained using backward elimination with a removal limit of $\mathrm{p}<0.10$. The full NPI was used in these analyses. . \# NPI item. 
Figure 1. Caregiver reported EQ5D utility in DLB and AD

$<$ Figure 1 here $>$

Interpretation of utility score: $1=$ =perfect health, $0=$ death 